\title{
The sensory basis of spatial memory in the rat
}

\author{
LUCIA ZOLADEK and WILLIAM A. ROBERTS \\ The University of Western Ontario, London, Ontario, Canada N6A 5C2
}

\begin{abstract}
Rats were given three stages of training on an eight-arm, elevated radial maze with food reward at the end of each arm. In Stage 1, rats were allowed to choose freely among the arms from the beginning of a trial. In Stage 2, three initial forced choices were followed by a series of free choices. In Stage 3, the central platform of the maze was rotated with the rat on it between the initial forced choices and the free choices. Following testing on these three stages, the animals were divided into four groups and deprived of selected senses. One group was made blind, a second anosmic, a third blind and anosmic, and a fourth was left normal. The same three stages of testing that had been conducted preoperatively then were run again postoperatively. Throughout these tests, the possible use of auditory cues was tested by presenting white noise on alternate trials. Finally, two further tests were carried out, the multiple rotations test and the removal-replacement test. The results indicated that visual cues, but not olfactory or auditory cues, played a critical role in the rat's ability to avoid previously entered alleys. There was evidence also that rats used internal cues from kinesthetic and/or vestibular receptors when visual cues were absent.
\end{abstract}

Recent articles by Olton, Collison, and Werz (1977) and Olton and Samuelson (1976) reported a surprising ability of the rat to remember spatial locations it had visited previously. Rats were tested on an elevated maze which contained eight arms that radiated from a central platform, with food reward placed at the end of each arm. With a high degree of accuracy, rats tended to collect food from each of the eight arms without reentering arms already chosen. Apparently, some mechanism allows rats precisely to keep track of positions in space and discriminate between them on the basis of whether or not they have been visited in the recent past.

In this paper, an experiment is reported which attempted to specify what sensory modalities the rat might be using in performing this task. There seem to be a number of possibilities. Rats might be marking alleys by remembering extramaze cues associated with each alley entered. Early studies of elevated maze learning suggest that use of extramaze visual cues is of considerable importance. Deprivation of these cues by blinding rats led to marked decrements in rate of learning (Honzik, 1936; Tsang, 1934). Another possibility is that rats are leaving odor trails on maze arms and then avoiding entrance into arms containing an odor. Honzik (1936) reported that anosmic rats learned an elevated maze more slowly than normal rats, and more recent research has indicated that one factor responsible for spontaneous

Support for preparation of this paper was provided by Grant A7894 from the National Research Council of Canada. Address requests for reprints to William A. Roberts, Department of Psychology, The University of Western Ontario, London, Ontario, Canada N6A 5C2. alternation in rats is avoidance of an odor trail (Douglas, 1966b; Rosen \& Stein, 1969). Olton and Samuelson (1976) presented evidence which suggested that olfaction was not a critical factor in rats' performance on the eight-arm maze. Neither saturating the maze with a strong odor nor rotating the maze in the middle of a trial reduced the tendency to choose arms which pointed in directions not previously chosen. Although these results suggest that under normal conditions olfaction is not a critical factor, there remains the possibility that olfactory cues might be used if other senses are not available. For example, Honzik (1936) found that anosmia led to a considerably greater retardation of learning in blind rats than in sighted rats.

The use of auditory cues is another mechanism by which rats might achieve a high level of accuracy. It is possible that rats keep track of points in space with respect to sound sources which serve as reference points. Again, the possibility that auditory cues might not be of importance in sighted rats but could become critical in blind rats is suggested by Honzik's (1936) finding that deaf-sighted rats learned elevated mazes as rapidly as normal rats, but deaf-blind rats were significantly inferior to blind rats. Further evidence of the use of auditory cues in maze learning comes from reports of echolocation in rats (Riley \& Rosenzweig, 1957; Rosenzweig, Riley, \& Krech, 1955).

A last possibility is that rats form a spatial-memory map of alleys entered on the basis of kinesthetic and/ or vestibular receptors. Once again, the use of movement-produced cues might become of relatively greater importance when a more primary sense such as vision is removed. However, Douglas (1966b) con- 
cluded that position in space was the primary cue for spontaneous alternation in rats and that the source of this cue was vestibular sensitivity (Douglas, 1966a).

In the present experiment, rats were run through a series of tests on the eight-arm maze and then were subjected to loss of various sensory cues by both surgical and nonsurgical manipulations. The same series of tests then were run postoperatively to determine the effects of losing specific sensory cues and possible interactive effects of loss of cues. The possibility that normal rats or rats deprived of certain senses might use internal cues based on kinesthetic and/or vestibular receptors was tested at certain stages of the experiment by forcing animals to enter three alleys of the maze and then rotating them on the center platform before allowing free choices among all eight alleys.

\section{METHOD}

\section{Subjects}

The subjects were eight adult male hooded rats. They were reduced to $85 \%$ of free-feeding weight and maintained at that level throughout the experiment.

\section{Apparatus}

The apparatus used was an elevated, eight-arm radial maze consisting of a circular central platform, $35.5 \mathrm{~cm}$ in diameter, and eight identical arms, each $76 \mathrm{~cm}$ long $\times 9 \mathrm{~cm}$ wide. The arms extended outward from the central platform, and adjacent arms were separated by $45^{\circ}$ angles. The maze was painted flat gray and stood $63.5 \mathrm{~cm}$ above the floor. Each arm had a rectangular metal food cup, $7.5 \mathrm{~cm}$ long $\times 5 \mathrm{~cm}$ wide $\times 2.5 \mathrm{~cm}$ deep, fixed to its extreme peripheral end. Adjacent to the central platform, each alley contained a door frame within which was mounted a transparent acrylic door. When raised, each door left a space, $11 \mathrm{~cm}$ high $\times 9 \mathrm{~cm}$ wide, through which an animal could gain access to an arm. These doors could be raised or lowered by individually weighted strings attached to a control board situated $1.8 \mathrm{~m}$ from the central platform. The central platform was constructed so that it could be rotated manually, independently of the rest of the maze, by a pulley system extending from the underside of the platform to the control board.

The maze was situated in the center of a room, $3.8 \mathrm{~m}$ long $x$ $3.2 \mathrm{~m}$ wide, which contained a counter along one wall, two windows, and a single door. Also in the room were a small writing table, two high-back stools, and a wastepaper basket. Illumination was provided by two enclosed, overhead fluorescent lighting units.

A Grason-Stadler white-noise generator, Model 901-B, was located on the counter top, and a speaker was suspended $91 \mathrm{~cm}$ above the central platform of the maze during postoperative stages of testing.

\section{Procedure}

All rats initially were gentled by handling for 1 week. The subjects then were placed on the eight-arm maze individually and allowed to explore the apparatus with all doors open for $30 \mathrm{~min}$. The food cups at the end of each arm were baited with two $45-\mathrm{mg}$ Noyes pellets for this and all subsequent phases of the experiment.

Following the familiarization period, Stage 1 of preoperative testing began. The animals were tested on two free-choice trials each day. The subject was placed on the maze facing a randomly chosen arm, and all doors were opened simultaneously. A trial consisted of 10 choices, and a choice was defined as a rat pro- ceeding to the end of an arm. Upon completion of each trial, the doors were lowered and the animal was returned to its home cage. The animals were tested in rotation, so that approximately $1 \mathrm{~h}$ elapsed between daily trials. Stage 1 training continued for each animal until its performance appeared to reach asymptotic level over four trials. The number of trials run varied between animals, with a range of 10 to 21 trials and a mean of 14.75 .

In Preoperative Stage 2, two trials with forced choices were carried out each day for a total of 3 days. On each trial, an animal was placed on the central platform with doors to all alleys closed. Three different doors then were opened and closed in succession, with the rat allowed to enter and return from each alley during the period that its door was open. Following the third forced choice, all eight doors were opened simultaneously, and the animal was allowed to make seven free choices. On the initial three forced choices, an animal was forced to enter alleys separated by $45^{\circ}$ (adjacent alleys), $90^{\circ}$ (a one-alley separation), or $135^{\circ}$ (a two-alley separation). Each degree of separation was used on two trials, and the direction in which the animal proceeded through these forced choices was clockwise on each of the two trials and counterclockwise on the other trial. The order in which these conditions were tested varied randomly between subjects.

Preoperative Stage 3 used the same procedures as Preoperative Stage 2, except that animals were tested for 10 trials and were rotated between forced and free choices. Following forced choices, the central platform and the animal upon it were rotated $0^{\circ}, 90^{\circ}$, $180^{\circ}, 270^{\circ}$, or $360^{\circ}$. There were two trials at each degree of rotation, one with rotation in a clockwise direction and the other in a counterclockwise direction. On $0^{\circ}$ rotation trials, the subject was delayed for approximately $3 \mathrm{sec}$ between forced and free choices.

At the conclusion of Preoperative Stage 3, the eight subjects were divided into four groups of two animals each. Based upon performance in Stages 2 and 3, these animals were matched into groups which assured approximately equal levels of mean accuracy in each group. These four groups then were assigned randomly the designations blind group, anosmic group, blind-anosmic group, and normal group. The blind and blind-anosmic groups were blinded by enucleation. Subsequent to this operation, the anosmic and blind-anosmic animals were made peripherally anosmic by bathing the olfactory mucosa with a $5 \%$ zinc sulfate solution. Details of this procedure can be found in Alberts and Galef (1971).

As a test for anosmia, an animal was placed in a wooden box measuring $66 \mathrm{~cm}$ long $\times 23 \mathrm{~cm}$ wide $\times 18 \mathrm{~cm}$ high, painted flat gray with a transparent acrylic lid. A small piece of Christie's Fudgee-O cookie was buried within a bedding of cork chips placed on the bottom of the box. The measure of olfactory sensitivity was the length of time that elapsed between placement in the box and discovery of the piece of cookie, as indicated by the animal grasping it with teeth or forepaws. A trial was ended after $5 \mathrm{~min}$ if the bit of cookie was not found. Following enucleation of rats assigned to blindness conditions, each rat in all four groups was tested for six trials, one per day, on the olfactory sensitivity test prior to zinc sulfate treatment in order to establish a baseline. After animals assigned to anosmic groups were treated with zinc sulfate, all animals were tested each day on the olfactory sensitivity test throughout Postoperative Stages 1, 2, and 3. It was found that time on the olfactory sensitivity test increased substantially for animals treated with zinc sulfate. If the latency of an anosmic animal began to return to baseline during postoperative testing, a further zinc sulfate treatment was administered.

Postoperative testing was carried out according to the same procedures used in preoperative testing, with 6 trials given on each of Stages 1 and 2 and 10 trials on Stage 3. The one new variable introduced in postoperative testing was white noise. A speaker placed over the central platform of the maze presented white noise (71 dB, re $20 \mu \mathrm{N} / \mathrm{m}^{2}$ ) on half of the trials in each stage and was turned off on the other half of the trials. For one animal in each group, white noise was presented on the first trial of a day 
and was absent on the second trial of the day. This order was reversed for the other animal of each group.

Following Stage 3 of postoperative testing, the animals were tested for six trials each on a multiple rotations test and a removalreplacement test. The multiple rotations test followed the same procedure as Stage 3, except that following the third forced choice, an animal was given three $360^{\circ}$ rotations in one direction followed by two and one-half rotations in the opposite direction. For each animal, the initial direction of rotation was clockwise on half of the trials and counterclockwise on the other half. In the removal-replacement test, an animal was removed from the apparatus after three forced choices and returned to its home cage in an adjacent room for $60 \mathrm{sec}$. It then was returned to the central platform and placed in a position facing $180^{\circ}$ away from the direction it had been facing when removed from the apparatus. Throughout these latter sets of tests, white noise was present on all trials.

\section{RESULTS}

\section{Preoperative Stages}

In Table 1 , the mean number of different alleys entered in the first eight choices is presented for each group in Stages 1, 2, and 3. It should be pointed out that the first three choices had to be correct in Stages 2 and 3 , since the animals were forced to enter different alleys. However, in the free-choice procedure used in Stage 1, the animals rarely made errors on the first three choices. The Stage 1 scores represent performance over the last 4 days of testing. Although the blind and normal groups are somewhat lower than the anosmic and blind-anosmic groups, the four groups do not differ significantly among one another, $F(3,4)=5.67, p>.05$. Performance in Stages 2 and 3 was highly accurate in all four groups, and there were no significant differences among groups in either stage, $F<1.0$ in both stages.

Further analyses indicated that the number of degrees of separation between forced choices had no effect on performance in Stages 2 and 3, F $<1.0$ in both stages. In Stage 3, it was found that neither the number of degrees of rotation between free and forced turns nor the direction of rotation had significant effects on performance, $F<1.0$ for both effects. In general, all eight rats showed a high degree of accuracy in choosing previously unentered alleys under a variety of forced choices and degrees and direction of rotation.

\section{Olfactory Sensitivity Test}

Mean latency of food discovery is shown for each group in Figure 1, with the darkened bars representing

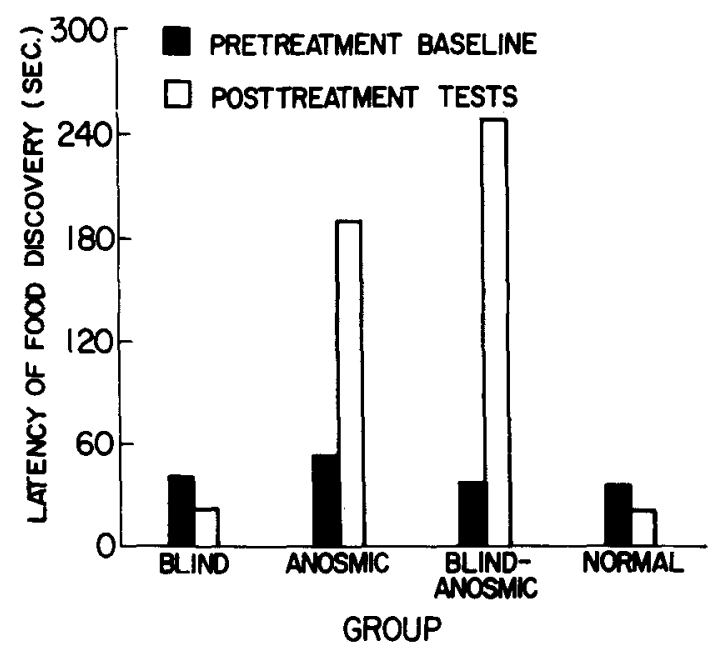

Figure 1. Mean latency of food discovery on pretreatment baseline trials and on posttreatment with zinc sulfate trials.

mean time on the 6 days of baseline testing and the unfilled bars indicating mean time over the 11 days of testing on Stages 1, 2, and 3. Mean latencies did not differ among groups on baseline testing, $F<1.0$. Following the zinc sulfate treatment, the anosmic and blind-anosmic groups showed a marked increase in latency, whereas the blind and normal groups dropped somewhat below baseline level. A blindness (blind vs. sighted animals) by anosmia (animals treated with zinc sulfate vs. untreated animals) analysis of variance on postoperative latencies showed a significant effect of the anosmia treatment, $F(1,4)$ $=137.56, p<.01$, but no significant difference between blind and sighted animals, $F(1,4)=3.50$, $\mathrm{p}>.05$, and no significant interaction of Anosmia by Blindness, $F(1,4)=2.94, p>.05$.

\section{Postoperative Stages}

Table 1 indicates that postoperative performance over the six trials of Stage 1 was nearly perfect for anosmic and normal groups, whereas the blind group was somewhat less accurate and the blind-anosmic group was substantially lower. Averaged over all four groups, it was found that mean number of different alleys entered on white-noise trials was 7.3 and the mean on trials without white noise was 7.4. Statistical analysis indicated that the difference between blind and sighted animals was marginally significant, $F(1,4)=7.41, p=.06$, and that there was no significant effect of anosmia, $F(1,4)=4.17$,

Table 1

Mean Number of Correct Choices (Different Alleys Entered in First Eight Choices)

\begin{tabular}{|c|c|c|c|c|c|c|c|c|}
\hline \multirow[b]{2}{*}{ Group } & \multicolumn{3}{|c|}{ Preoperative Stage } & \multicolumn{3}{|c|}{ Postoperative Stage } & \multirow{2}{*}{$\begin{array}{c}\text { Multiple } \\
\text { Rotations }\end{array}$} & \multirow{2}{*}{$\begin{array}{c}\text { Removal- } \\
\text { Replacement }\end{array}$} \\
\hline & 1 & 2 & 3 & 1 & 2 & 3 & & \\
\hline Blind & 7.1 & 7.7 & 7.7 & 7.5 & 6.6 & 6.7 & 6.0 & 6.2 \\
\hline Anosmic & 7.9 & 7.9 & 7.9 & 7.8 & 7.8 & 7.6 & 7.8 & 7.8 \\
\hline Blind-Anosmic & 7.9 & 7.9 & 7.9 & 6.3 & 6.8 & 6.4 & 5.9 & 6.0 \\
\hline Normal & 6.9 & 7.9 & 7.9 & 8.0 & 7.9 & 7.8 & 7.8 & 7.8 \\
\hline
\end{tabular}


$\mathrm{p}>.05$, or of white noise, $\mathrm{F}<1.0$.

To further examine the effects of blindness on performance, percentage of correct choices is plotted against ordinal position of choice for blind and sighted subjects in Figure 2. The curves indicate that blind animals were equally as accurate as sighted animals up to the fifth choice but dropped progressively below sighted animals on Choices 6 through 8 . Analysis of variance indicated a nonsignificant effect of blindness, $F(1,6)=4.52$, $p>.05$, but significant effects of position of choice, $F(7,42)=$ $5.48, \mathrm{p}<.01$, and of the Blindness by Choice interaction, $F(7,42)=3.92, p<.01$. When comparisons of blind and sighted animals at each choice were made, the only significant difference was at Choice 8, $\mathrm{t}(6)=3.68, \mathrm{p}<.01$.

The inferior performance of blinded animals became more apparent in Postoperative Stages 2 and 3. Anosmic and normal animals performed close to a perfect score of 8.0, and blind and blind-anosmic animals were about an entire error lower in accuracy. Once again, the differences between trials with and without white noise were negligible. The effect of blindness was significant in both Stage $2, F(1,4)=$ $327.22, p<.01$, and Stage 3, $F(1,4)=17.63$, $p<.05$. The effects of anosmia and of white noise did not approach significance in either stage. As in preoperative tests, different patterns of forced choices and different degrees and directions of rotation had no significant effects on performance.

\section{Additional Tests}

It is clear from Postoperative Stages 1, 2, and 3 that visual cues played an important role in the rat's ability to avoid previously entered arms. It was

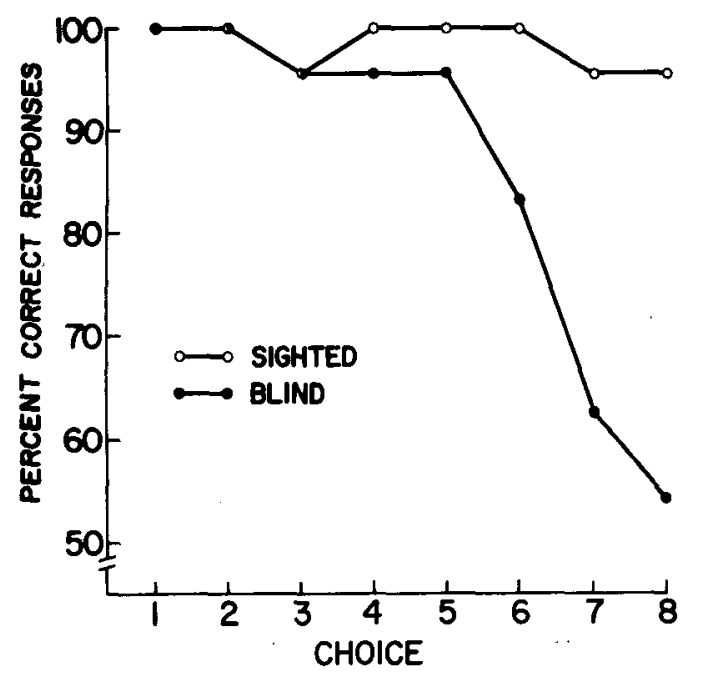

Figure 2. Percentage of correct choices for blind and sighted animals on Postoperative Stage 1, plotted as a function of ordinal position of choice. expected that rotating the central platform on Stage 3 might further hinder the performance of blind rats, if these animals were dependent upon internal cues which keep track of the position of the body in space. However, blind animals showed no substantial reduction in performance from Stage 2 to Stage 3, and degree of rotation had no effect on blind subjects. It is possible that blind animals were able to make adjustments in their choices which took into account these rotations. The multiple rotations test was carried out to test the hypothesis that more extensive rotation might make such adjustments very difficult and thus lower the performance of blind animals. The column for the multiple rotations test in Table 1 shows that sighted animals were unaffected by multiple rotations, but blind animals now were significantly less accurate than on Stage 3 of postoperative testing, $t(3)=4.60$, $\mathrm{p}<.01$.

The removal-replacement test was carried out as a further attempt to disrupt the animals' sense of spatial position. The removal-replacement column of Table 1 shows that once again sighted animals were unaffected, but blind animals were significantly less accurate than on Stage 3, $\mathrm{t}(3)=2.50, \mathrm{p}<.05$.

\section{DISCUSSION}

The results of Preoperative Stages 1, 2, and 3 verified the findings of Olton et al. (1977) and Olton and Samuelson (1976). That is, animals were highly accurate in avoiding reentry into alleys already visited both in the completely free-choice situation of Stage 1 and in the free choices that followed forced choices in Stages 2 and 3. Postoperative tests on Stages 1, 2, and 3 suggested that the primary cues used by animals in this experiment were visual in nature. Animals which were blinded showed a clear loss of accuracy, whereas animals made anosmic continued to perform at the high level achieved on preoperative stages. The introduction of white noise to mask potential auditory cues had no effect at all on performance.

The importance of visual cues discovered here must be qualified. The finding that rats were primarily dependent upon visual cues in this experiment and perhaps also in Olton's experiments does not rule out the possibility that other senses might become of major importance in other environments. No specific auditory cues or odor cues were provided in this experiment, but an environment that was clearly differentiated visually was provided. If a constant sound source were made available or the alleys were covered with substances that provided different odors, then evidence for the use of auditory or olfactory cues might be revealed. This might be particularly the case if the opportunity to use visual cues were reduced by using a visually homogeneous 
environment surrounding the maze. On the other hand, it is possible that the ability of rats to continually choose novel directions in their environment is constrained to use of visual cues. Only further research which systematically varies sensory modalities and environmental cues will answer this question.

There is some evidence in this report to suggest that vision was not the only sense by which rats were finding their way around the eight-arm maze. Blind rats may have been making use of internal vestibular and/or kinesthetic cues to keep track of positions in space. An examination of the errors made by blind rats on Postoperative Stages 2 and 3 indicates that only $15.6 \%$ of errors on Stage 2 and $10.2 \%$ of errors on Stage 3 were repetitions of alleys entered on free choices. This finding was extremely pronounced on the multiple-rotations and removal-replacement tests; of a total of 82 errors made by blind animals on these tests, only one error was a repetition of a freely chosen alley. In other words, almost all errors were repetitions of alleys which animals were forced to enter. These observations indicate that over the first five free choices blind animals made after forced choices, they were very accurately avoiding repeating recently made choices. The same point is made in Figure 2, where it can be observed that blind and sighted animals were about equally accurate over the first five choices in Stage 1 of postoperative testing. Only on the last three choices did blind animals become progressively less accurate in avoiding previously entered alleys. It may be that internal cues serve to allow an animal to make gross discriminations between positions in space. However, finer discriminations required when only one or two alleys have been unentered may be difficult to make without the benefit of visual cues.

Further evidence that blind rats used internal cues on Postoperative Stages 1, 2, and 3 is found in performance on the multiple rotations and removalreplacement tests. Both of these tests involved manipulations between free and forced choices which were designed to completely disorient the rat spatially. In both tests, accuracy decreased significantly for blind animals but was unaffected in sighted animals.

In summary, these results suggest that rats in this experiment primarily used visual cues to achieve a high level of accuracy. Internal cues supplied by kinesthetic and/or vestibular receptors also may have been used for this purpose and may have become of particular importance in animals deprived of vision. However, internal cues about the position of the body in space may not allow an animal to make as precise discriminations between spatial points as is the case with visual cues. It is interesting to note that the conclusions reached from this study are almost identical to those of Beritoff (1965). Using dogs and cats, Beritoff examined the effects of loss of selected senses on spatial orientation. It was concluded that the most significant cues for orientation in space were those supplied by the visual and vestibular receptors.

\section{REFERENCES}

Alberts, J. R., \& Galef, B. G., JR. Acute anosmia in the rat: A behavioral test of a peripherally-induced olfactory deficit. Physiology and Behavior, 1971, 6, 619-621.

BERITOFF, J. S. [Neural mechanisms of higher vertebrate behavior] (W. T. Liberson, Ed. and trans.). Boston: Little, Brown, 1965.

Douglas, R. J. Spontaneous alternation and middle ear disease. Psychonomic Science, 1966, 4, 243-244. (a)

Douglas, R. J. Cues for spontaneous alternation. Journal of Comparative and Physiological Psychology, 1966, 62, 171-183. (b)

HonzIK, C. H. The sensory basis of maze learning in rats. Comparative Psychology Monographs, 1936, 13(Whole No. 4).

Olton, D. S., \& SAmuelson, R. J. Remembrance of places passed: Spatial memory in rats. Journal of Experimental Psychology: Animal Behavior Processes, 1976, 2, 97-116.

Olton, D. S., Collison, C., \& Werz, M. A. Spatial memory and radial arm maze performance of rats. Learning and Motivation, 1977, 8, 289-314.

RILEY, D. A., \& RoseNzWEIG, M. R. Echolocation in rats. Journal of Comparative and Physiological Psychology, 1957, 50, 323-328.

Rosen, J. J., \& Stein, D. G. Spontaneous alternation behavior in the rat. Journal of Comparative and Physiological Psychology, 1969, 68, 420-426.

Rosenzweig, M. R., RILEY, D., \& KRECh, D. Evidence for echolocation in rats. Science, $1955,121,600$.

TsANG, Y. G. The functions of the visual area of the cerebral cortex of the rat in the learning and retention of the maze. I. Comparative Psychology Monographs, 1934, 10(Whole No. 4).

(Received for publication June 21, 1977; accepted October 25, 1977.) 